\title{
Estimating fusion properties for functionalised acids
}

\author{
S. Compernolle, K. Ceulemans, and J.-F. Müller \\ Belgian Institute for Space-aeronomy, Ringlaan 3, 1180 Brussels, Belgium \\ Received: 17 November 2011 - Published in Atmos. Chem. Phys. Discuss.: 4 March 2011 \\ Revised: 29 June 2011 - Accepted: 4 August 2011 - Published: 17 August 2011
}

\begin{abstract}
Multicomponent organic aerosol (OA) is likely to be liquid, or partially liquid. Hence, to describe the partitioning of these components, their liquid vapour pressure is desired. Functionalised acids (e.g. diacids) can be a significant part of OA. But often measurements are available only for solid state vapour pressure, which can differ by orders of magnitude from their liquid counterparts. To convert such a sublimation pressure to a subcooled liquid vapour pressure, fusion properties (two out of these three quantities: fusion enthalpy, fusion entropy, fusion temperature) are required. Unfortunately, experimental knowledge of fusion properties is sometimes missing in part or completely, hence an estimation method is required. Several fusion data estimation methods are tested here against experimental data of functionalised acids, and a simple estimation method is developed, specifically for this family of compounds, with a significantly smaller estimation error than the literature methods.
\end{abstract}

\section{Introduction}

Diacids can be a significant part of OA, according to both field measurements and smog chamber experiments (Limbeck et al., 2001; Baboukas et al., 2000; Claeys et al., 2007; Yu et al., 1999). Due to the multicomponent nature of OA it is often glassy or liquid-like at ambient temperature even if the individual components are crystalline solids when in pure state, as was recently demonstrated for a mixture of diacids (Cappa et al., 2008b). To describe the partitioning of a compound to the aerosol, its liquid vapour pressure is required. Vapour pressures of polyacids have been measured over sev-

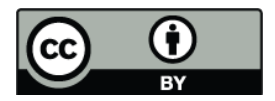

Correspondence to: S. Compernolle (steven.compernolle@aeronomie.be) eral decades (Bradley and Cotson, 1953; Arshadi, 1974) but recently work in this area has intensified, with several publications in the last two years (Booth et al., 2010, 2011; Frosch et al., 2010; Soonsin et al., 2010; Pope et al., 2010).

Unfortunately, most pure diacids are stable as solid at ambient temperature, although a liquid metastable state can also occur. To obtain the liquid vapour pressure, one could extrapolate from measurements above the melting point $T_{\text {fus }}$. As $T_{\text {fus }}$ can be a few hundred Kelvin above the temperature of interest, this approach is prone to error, although there are also examples of excellent agreement, e.g. for succinic acid (Riipinen et al., 2006). Some groups have measured, at ambient temperature, the vapour pressure of the liquid diacid in water/diacid droplet particles, using the electrodynamic balance (EDB) (Zardini et al., 2006; Pope et al., 2010; Soonsin et al., 2010) or the tandem differential mobility analyzer (TDMA) techniques (Riipinen et al., 2007; Koponen et al., 2007). In such cases, the activity coefficient is also needed in order to determine the vapour pressure of the pure diacid, which can be calculated using empirical methods (Peng et al., 2001; Hansen et al., 1991). Other groups have measured, at ambient temperature (or for Ribeiro da Silva et al. (2000, 2001), at least below $T_{\text {fus }}$ ), the vapour pressure of the solid using temperature programmed desorption (Cappa et al., 2007, 2008a), TDMA (Frosch et al., 2010; Salo et al., 2010; Bilde and Pandis, 2001; Bilde et al., 2003) or EDB (Soonsin et al., 2010) on aerosol particles and Knudsen effusion on bulk samples (Ribeiro da Silva et al., 2000, 2001; Booth et al., 2010, 2011). However, there can be orders of magnitude difference between measurements of different groups for the same compound (e.g. for sebacic acid, 3 orders of magnitude between Cappa et al., 2007 and Salo et al., 2010), way above the reported experimental errors (typically $30-50 \%$ ). It has been speculated that this might be due to the experimental technique employed (Cappa et al., 2007; Pope et al., 2010) or to

Published by Copernicus Publications on behalf of the European Geosciences Union. 
the physical nature of the diacids (Zardini et al., 2006; Soonsin et al., 2010; Salo et al., 2010) (presence of defects; partially or completely liquid/amorphous character). Soonsin et al. (2010) have measured subcooled liquid vapour pressures of mixtures with only a very small water content, and vapour pressures of the saturated solution, which allows the derivation of the vapour pressure of the pure liquid and solid, respectively.

Booth et al. (2010, 2011) measured sublimation pressures of several diacids, converted them to subcooled liquid vapour pressures using fusion data, and then compared them to several methods that estimate liquid vapour pressure from molecular structure. The conclusion was that none of these methods performed well.

Even if a given experimental sublimation pressure can be considered accurate, one still needs fusion data (fusion temperature $T_{\text {fus }}$, enthalpy $\Delta H_{\text {fus }}$ and entropy $\Delta S_{\text {fus }}$ ) to obtain a subcooled liquid vapour pressure (Prausnitz et al., 1999), but it occurs often that fusion data is unavailable, or only the fusion temperature is known (Ribeiro da Silva et al., 2000; Monster et al., 2004; Frosch et al., 2010). General estimation methods for $T_{\text {fus }}, \Delta H_{\text {fus }}$ (Joback and Reid, 1987; Marrero and Gani, 2001; Zhao and Yalkowsky, 1999) or $\Delta S_{\text {fus }}$ (Myrdal and Yalkowsky, 1997; Jain et al., 2004a) give significant errors for functionalised acids, as we will show below. Therefore, a simple method is developed in this work to estimate the fusion properties of $\mathrm{C}_{2}-\mathrm{C}_{10}$ saturated acids, with one or more other functional groups: hydroxyl, carbonyl and/or acid. This method is then compared with methods of the literature.

\section{Literature data}

In Table S1 of the Supplement, we present experimental fusion data for functionalised acids. Important data sources were Linstrom and Mallard (NIST), Acree and Chickos (2010), Booth et al. (2010, 2011), Lide (2000) and Aldrich (1990). In those cases where solid-solid transitions were present the sum over all fusion data was taken:

$$
\begin{aligned}
\Delta H_{\text {fus }, \text { tot }} & =\sum_{i} \Delta H_{\text {trans }, \mathrm{i}} \\
T_{\text {fus, tot }} & =\frac{\Delta H_{\text {fus }, \text { tot }}}{\Delta S_{\text {fus }, \text { tot }}}
\end{aligned}
$$

In many cases, only $T_{\text {fus }}$ was available, not $\Delta H_{\text {fus. }}$. For example, $\Delta H_{\text {fus }}$ was measured for only a small minority of the compounds in our dataset with one acid functionality. This is an obvious obstacle to model development.

Whenever possible, we included per compound multiple reference sources. If different sources agreed, only one was taken into account, and prevalence was given to the case where both $T_{\text {fus }}$ and $\Delta H_{\text {fus }}$ are available. In a few instances, quite different $T_{\text {fus }}$ data points or $T_{\text {fus }}, \Delta H_{\text {fus }}$ data point couples were found for a compound with the same, non-stereospecific, structural formula. Sometimes this could clearly be ascribed to the fact that the compounds were different stereo-isomers, but of course also experimental uncertainty could play a role. These data points were then all taken into account.

For oxalic acid, $T_{\text {fus }}$ reported by Booth et al. (2010) conflicts with the transition data reported by NIST and Thalladi et al. (2000). It is possible that Booth et al. (2010) found a solid-solid transition point rather than a fusion point (A. M. Booth, personal communication, 2010). However, interpretation of experimental data is hampered due to uncertainty regarding the precise structure of the solid oxalic acid (Soonsin et al., 2010). Therefore, we exclude oxalic acid in our comparison analysis of experimental with modeled data.

\section{Solid to liquid conversion: impact of heat capacity difference}

A subcooled liquid vapour pressure $p_{l}^{0}$ is not directly experimentally accessible, unless a metastable liquid state can be prepared. We are interested in the factor necessary to convert sublimation pressure to subcooled liquid vapour pressure,

$\omega_{l s}(T) \equiv \log _{10} \frac{p_{l}^{0}(T)}{p_{s}^{0}(T)}=\frac{\Delta H_{\text {fus }}(T)}{\ln (10) R T}-\frac{\Delta S_{\text {fus }}(T)}{\ln (10) R}$

with $\Delta H_{\text {fus }}(T), \Delta S_{\text {fus }}(T)^{1}$ the enthalpy, entropy difference between subcooled liquid and solid state respectively at temperature $T$. These can be found from knowledge of the fusion data at the triple point $T_{\text {tr }}, \Delta H_{\text {fus }}\left(T_{\text {tr }}\right), \Delta S_{\text {fus }}\left(T_{\text {tr }}\right)=$ $\frac{\Delta H_{\text {fus }}\left(T_{\text {tr }}\right)}{T_{\text {tr }}}$, and the heat capacity $C_{p}$ of both solid and subcooled liquid in the temperature region $\left[T, T_{\mathrm{tr}}\right]$ (e.g. Prausnitz et al., 1999).

$$
\begin{aligned}
\Delta H_{\text {fus }}(T)= & \Delta H_{\text {fus }}\left(T_{\text {tr }}\right)+ \\
& \int_{T_{\text {tr }}}^{T}\left(C_{p, l}\left(T^{\prime}\right)-C_{p, s}\left(T^{\prime}\right)\right) d T^{\prime} \\
\Delta S_{\text {fus }}(T)= & \Delta S_{\text {fus }}\left(T_{\text {tr }}\right)+ \\
& \int_{T_{\text {tr }}}^{T} \frac{\left(C_{p, l}\left(T^{\prime}\right)-C_{p, s}\left(T^{\prime}\right)\right)}{T} d T^{\prime}
\end{aligned}
$$

$T_{\text {tr }}$ is generally unavailable for the compounds of our interest, but replacement with the fusion temperature $T_{\text {fus }}$ incurs little error (Prausnitz et al., 1999). As $C_{p, l}(T)$ is not accessible below the triple point, one possible solution is to extrapolate it. Another common approximation is to assume that the

\footnotetext{
${ }^{1}$ Note that we indicate the temperature dependence of $\Delta H_{\text {fus }}, \Delta S_{\text {fus }}$ only in this section. In the other sections it is always assumed that they are taken at $T_{\text {fus }}$.
} 
difference $\Delta C_{p, l s}=C_{p, l}-C_{p, s}$ is temperature-independent:

$$
\Delta H_{\text {fus }}(T) \approx \Delta H_{\text {fus }}\left(T_{\text {fus }}\right)+\Delta C_{p, l s}\left(T-T_{\text {fus }}\right)
$$

$$
\Delta S_{\text {fus }}(T) \approx \Delta S_{\text {fus }}\left(T_{\text {fus }}\right)+\Delta C_{p, l s} \ln \frac{T}{T_{\text {fus }}}
$$

where $\Delta C_{p, l s}$ is taken at the fusion point. Note that $C_{p, s}, C_{p, l}$ at $T_{\text {fus }}$ become infinite so they have to be obtained by extrapolation from temperatures below and above $T_{\text {fus }}$ respectively (see e.g. Neau et al., 1997). In our case, the temperature of interest can be hundreds of Kelvin below the fusion point, but as $C_{p, l}(T)$ below $T_{\text {fus }}$ is inaccessible, it is difficult to assess the error incurred by approximations (6) and (7).

Unfortunately, for the compounds of our interest, a measured $\Delta C_{p, l s}$ is typically unavailable. Common approximations are (Grant et al., 1984)

$$
\Delta C_{p, l s} T \ll \Delta H_{\text {fus }}\left(T_{\text {fus }}\right)-\Delta C_{p, l s} T_{\text {fus }} \Rightarrow \Delta C_{p, l s} \approx 0
$$

$$
\begin{gathered}
\Delta C_{p, l s} T \gg \Delta H_{\text {fus }}\left(T_{\text {fus }}\right)-\Delta C_{p, l s} T_{\text {fus }} \Rightarrow \\
\Delta C_{p, l s} \approx \Delta S_{\text {fus }}\left(T_{\text {fus }}\right)
\end{gathered}
$$

Also the intermediate approximation, $\Delta C_{p, l s} \approx$ $0.5 \Delta S_{\text {fus }}\left(T_{\text {fus }}\right)$ can be taken (Tsonopoulos, 1970). In Fig. 1 the sensitivity of $\omega_{l s}(T)$ on the two extreme approximations for $\Delta C_{p, l s}$ (Eqs. 8 and 9) is explored. $\log _{10} \frac{p_{l}^{0}}{p_{s}^{0}}$ at $298 \mathrm{~K}$ differs for glutaric acid only by 0.08 (a factor 1.2 for $\frac{p_{l}^{0}}{p_{s}^{0}}$ ) between both assumptions, by 0.38 for succinic acid (a factor 2.4) and by 0.91 for tartaric acid (a factor 8.2). While for a compound such as tartaric acid the choice for $\Delta C_{p, l s}$ is certainly relevant, for most compounds in our dataset where both $T_{\text {fus }}, \Delta S_{\text {fus }}$ are known, $\log _{10} \frac{p_{l}^{0}}{p_{s}^{0}}$ differs by 0.3 or less between both extreme assumptions. This is often within the uncertainty on $\log _{10} p_{s}^{0}$.

Chickos (2003) present a group contribution method to estimate $C_{p, l}, C_{p, s}$ at $298.15 \mathrm{~K}$. As we assume that $\Delta C_{p, l s}$ is temperature-independent, we could set

$$
\begin{aligned}
\Delta C_{p, l s} & \approx \Delta C_{p, l s}(298.15 \mathrm{~K}) \\
& \approx C_{p, l}^{\text {Chickos }}(298.15 \mathrm{~K})-C_{p, s}^{\text {Chickos }}(298.15 \mathrm{~K})
\end{aligned}
$$

Chickos (2003) themselves however recommend using

$$
\begin{aligned}
\Delta C_{p, l s} \approx & \left(10.58 \frac{\mathrm{J}}{\mathrm{molK}}+0.26 C_{p, l}^{\text {Chickos }}(298.15 \mathrm{~K})\right) \\
& -\left(0.75 \frac{\mathrm{J}}{\mathrm{molK}}+0.15 C_{p, s}^{\text {Chickos }}(298.15 \mathrm{~K})\right)
\end{aligned}
$$

In Fig. 2 both approximations (called Chickos 1 and 2) are compared vs. the approximation $\Delta C_{p, l s}=\Delta S_{\text {fus }}\left(T_{\text {fus }}\right)$. The

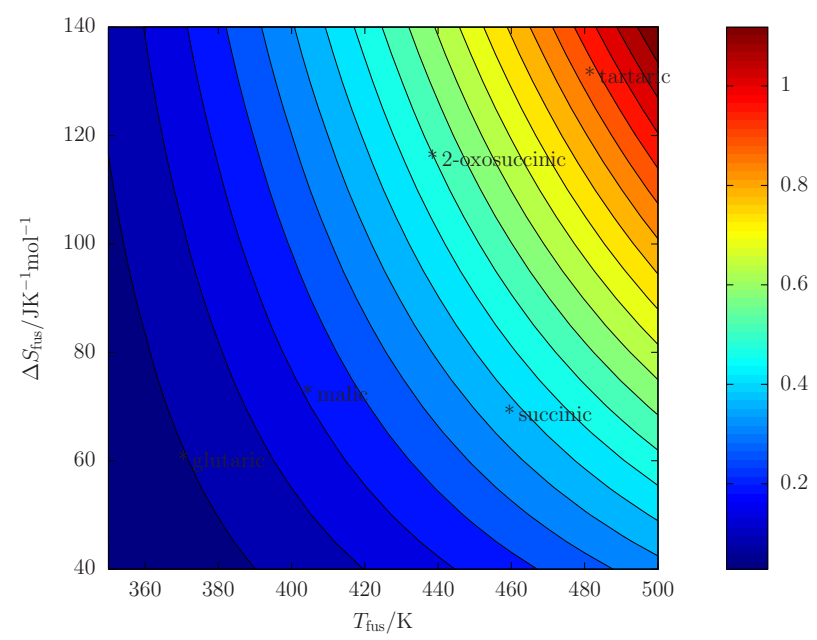

Fig. 1. $\omega_{l s}\left(\Delta C_{p, l s}=0\right)-\omega_{l s}\left(\Delta C_{p, l s}=\Delta S_{\text {fus }}\left(T_{\text {fus }}\right)\right)$ at $298 \mathrm{~K}$ vs. $T_{\text {fus }}$ and $\Delta S_{\text {fus }}\left(T_{\text {fus }}\right)$. The position of some diacids is indicated.

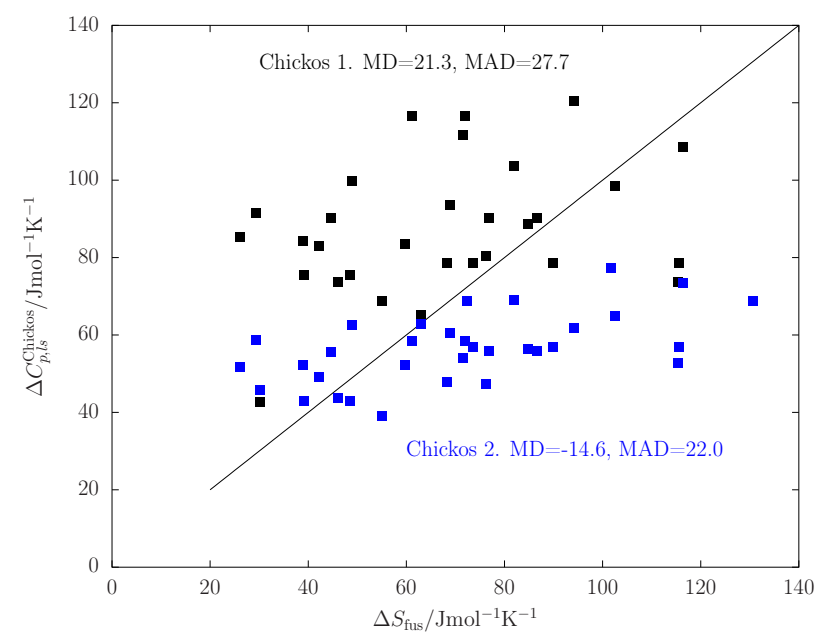

Fig. 2. $\Delta C_{p, l s}$ as estimated by the method of Chickos (2003) (Chickos 1 (black) and 2 (blue): see text for explanation) vs. $\Delta C_{p, l s}$ as approximated by $\Delta S_{\text {fus }}\left(T_{\text {fus }}\right)$ for the compounds in our data set. The 1:1 diagonal is indicated (black line). The mean deviation and mean absolute deviation are also given.

mean deviation (MD) and mean absolute deviation (MAD), defined as

$$
\mathrm{MD}=\frac{1}{N} \sum_{i}^{N}\left(f_{i}^{\mathrm{est}}-f_{i}^{\mathrm{exp}}\right)
$$

$\mathrm{MAD}=\frac{1}{N} \sum_{i}^{N}\left|f_{i}^{\mathrm{est}}-f_{i}^{\mathrm{exp}}\right|$

where $f_{i}^{\text {exp }}$ is an experimental measurement and $f_{i}^{\text {est }}$ an estimation, are also given. It is clear that large discrepancies exist, both in terms of MD and MAD, between on 

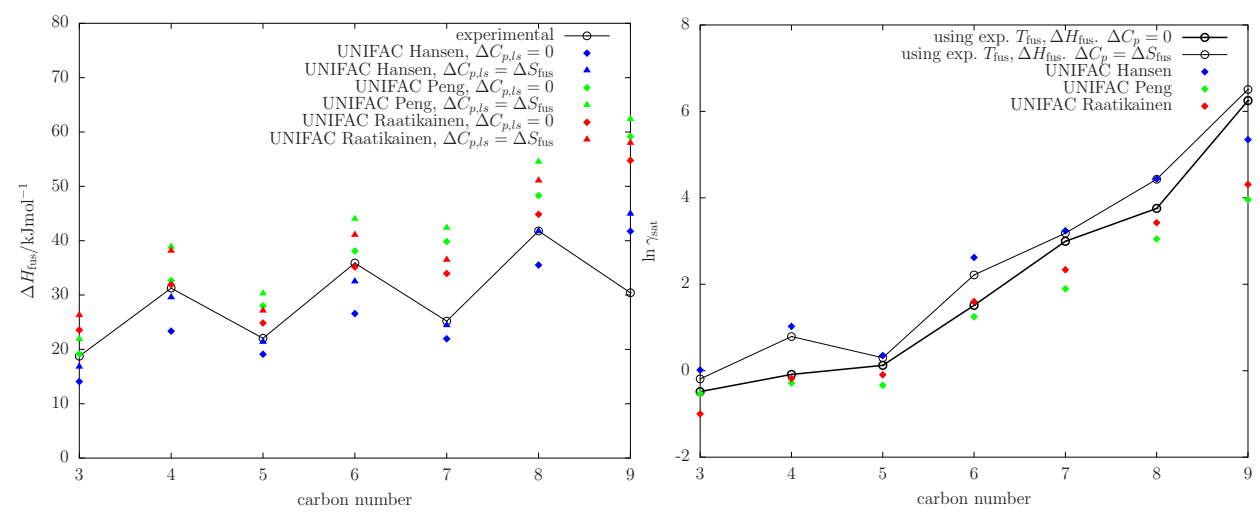

Fig. 3. Left: $\Delta H_{\text {fus }}$ for linear diacids (C3-C9), experimental or estimated from experimental $T_{\text {fus }}, x_{\text {sat }}$ and estimated $\gamma_{\text {sat }}$ (at $298 \mathrm{~K}$ ). Right: $\ln \gamma_{\text {sat }}$ for the same linear diacids, calculated from experimental $T_{\text {fus }}, \Delta H_{\text {fus }}$ data and the two limiting assumptions for $\Delta C_{p, l s}\left(0\right.$ to $\left.\Delta S_{\text {fus }}\right)$, or estimated from several UNIFAC methods.

the one hand the two assumptions involving the method of Chickos (2003), and on the other hand, the assumption of $\Delta C_{p, l s}=\Delta S_{\text {fus }}\left(T_{\text {fus }}\right)$. Equation (10) predicts $\Delta C_{p, l s}$ larger than $\Delta S_{\text {fus }}\left(T_{\text {fus }}\right)$ in most cases, while, under the assumption of a temperature-independent $\Delta C_{p, l s}$, this should not be possible. Hence Eq. (10) seems an invalid approximation for $\Delta C_{p, l s}$.

\section{Use of solubility to estimate fusion properties}

The vapour pressure of a compound at saturation is equal to its sublimation pressure as a pure solid (e.g. Prausnitz et al., 1999):

$p_{s}^{0}=\gamma_{\mathrm{sat}} x_{\mathrm{sat}} p_{l}^{0}$

with $x_{\text {sat }}$ the saturation mole fraction of the compound and $\gamma_{\text {sat }}$ the activity coefficient. Combination of Eqs. (3), (6), (7) results in

$$
\begin{aligned}
\ln \left(x_{\text {sat }} \gamma_{\text {sat }}\right)= & \frac{\Delta H_{\text {fus }}}{R}\left(\frac{1}{T_{\text {fus }}}-\frac{1}{T}\right) \\
& -\frac{\Delta C_{p, l s}}{R}\left(1-\frac{T_{\text {fus }}}{T}-\ln \left(\frac{T}{T_{\text {fus }}}\right)\right)
\end{aligned}
$$

Suppose, as is frequently the case, that $T_{\text {fus }}$ is known but $\Delta H_{\text {fus }}$ is not. Then $\Delta H_{\text {fus }}$ can be estimated from an experimental $x_{\text {sat }}$ (e.g. in water), an estimated $\gamma_{\text {sat }}$ (e.g. by UNIFAC: UNIQUAC Functional groups Activity Coefficients) and an approximation for $\Delta C_{p, l s}$ (from 0 to $\Delta S_{\text {fus }}$, see Sect. 3). Figure 3 compares experimental $\Delta H_{\text {fus }}$ to $\Delta H_{\text {fus }}$ calculated from Eq. (15) for linear diacids with 3-9 carbon atoms.

Saturation concentrations in water at $298.15 \mathrm{~K}$ were taken from Apelblat and Manzurola (1987, 1990); Lide (2000). $\gamma_{\text {sat }}$ was calculated by different parameterisations for UNIFAC: the original one from Fredenslund et al. (1975); Hansen et al. (1991) (UNIFAC-Hansen), and the more recent ones from Peng et al. (2001) (UNIFAC-Peng) and Raatikainen and Laaksonen (2005) (UNIFAC-Raatikainen). The parameterisation of UNIFAC-Hansen was developed from data mainly for simple monofunctional compounds. UNIFAC-Peng and UNIFAC-Raatikainen on the other hand, were specifically developed to describe the non-ideality of mixtures of water with diacids and multifunctional acids and should be more appropriate than UNIFAC-Hansen. UNIFAC-Peng is based on the water activity of such mixtures, while for UNIFACRaatikainen also solubility data was used.

From Fig. 3 it is clear that both UNIFAC-Peng and UNIFAC-Raatikainen overestimate $\Delta H_{\text {fus }}$ for the larger diacids (7-9 carbon atoms). These diacids were not included in the dataset for the derivation of the UNIFACPeng and UNIFAC-Raatikainen parameters. For 3-6 carbon atoms, the agreement of both methods with experiment is best if $\Delta C_{p, l s}=0$ is assumed. This can partly be understood because Raatikainen and Laaksonen (2005) derived the parameters for UNIFAC-Raatikainen, using solubility data and Eq. (15) with the assumption $\Delta C_{p, l s}=0$. Interestingly, UNIFAC-Hansen, combined with the assumption of $\Delta C_{p, l s}=\Delta S_{\text {fus }}$, has the best overall agreement. All three methods overestimate $\Delta H_{\text {fus }}$ for azelaic acid ( 9 carbon atoms). Also shown in Fig. 3 is the estimation of $\gamma_{\text {sat }}$ from the experimental $T_{\text {fus }}, \Delta H_{\text {fus }}$ data and the two limiting assumptions for $\Delta C_{p, l s}\left(0\right.$ to $\left.\Delta S_{\text {fus }}\right)$. The true activity coefficient should be between both limits. The underestimation of $\gamma_{\text {sat }}$ by UNIFAC-Peng and UNIFAC-Raatikainen for diacids with 7-9 carbon atoms is clear.

Given the uncertainty on $\Delta H_{\text {fus }}$ estimation with this approach, both due to the activity coefficient modelling and the assumption for $\Delta C_{p, l s}$, we decided not to pursue this route to obtain additional $\Delta H_{\text {fus }}$ data. 


\section{Development of a new estimation method}

The fusion data of linear diacids follow an even-odd alternation (Roux et al., 2005; Thalladi et al., 2000) in function of carbon number. Thalladi et al. (2000) could explain this behaviour through analysis of the crystal structure of linear diacids, which is particularly stable for even-numbered chains. Using the linear diacid data from Booth et al. (2010); Roux et al. (2005), excluding oxalic acid, one obtains

$$
\begin{aligned}
& \# \mathrm{CH}_{2} \text { odd: } \\
& \frac{\Delta H_{\text {fus }}\left(T_{\text {fus }}\right)}{\mathrm{kJ} \mathrm{mol}^{-1}}=16.464+1.909 \cdot \# \mathrm{CH}_{2}, R^{2}=0.984 \\
& \frac{\Delta S_{\text {fus }}\left(T_{\text {fus }}\right)}{\mathrm{J} \mathrm{K}^{-1} \mathrm{~mol}^{-1}}=40.91+5.81 \cdot \# \mathrm{CH}_{2}, R^{2}=0.995 \\
& \# \mathrm{CH}_{2} \quad \text { even: } \\
& \frac{\Delta H_{\text {fus }}\left(T_{\text {fus }}\right)}{\mathrm{kJ} \mathrm{mol}^{-1}}=25.704+2.657 \cdot \# \mathrm{CH}_{2}, R^{2}=0.998 \\
& \frac{\Delta S_{\text {fus }}\left(T_{\text {fus }}\right)}{\mathrm{J} \mathrm{K}^{-1} \mathrm{~mol}^{-1}}=52.45+8.11 \cdot \# \mathrm{CH}_{2}, R^{2}=0.999
\end{aligned}
$$

where $\# \mathrm{CH}_{2}$ denotes the number of methylene groups and $R^{2}$ the coefficient of determination. Using correlations (18) and (21), a $T_{\text {fus }}$ of $490 \mathrm{~K}$ would be predicted for oxalic acid, even higher than the fusion point reported by NIST and Thalladi et al. (2000). This gives further argument that the fusion point reported by Booth et al. (2010) could be a solid-solid transition point.

No such clear correlation of fusion enthalpy or entropy with carbon number exists for the nonlinear functionalised acids. For example, the cyclic diacid 1,3-cyclohexane dicarboxylic diacid has a lower $\Delta H_{\text {fus }}\left(T_{\text {fus }}\right)$ and $\Delta S_{\text {fus }}\left(T_{\text {fus }}\right)$ than 1,3-cyclopropane diacid. Instead, we use as independent variable the effective torsional bond number $\tau$ (Dannenfelser and Yalkowsky, 1996). This variable plays a similar role as the $\mathrm{CH}_{\mathrm{n}}$ groups in common group-contribution methods, as $\# \mathrm{CH}_{2}+\# \mathrm{CH}+\# \mathrm{C}$ is the mostly dominant contribution of the noncyclic carbon atoms to $\tau$. As interactions between functional groups are important both in the liquid and the solid phase, also the number of nonacid (carbonyl and hydroxy) and acid functional groups is taken into account. Finally, the identification of the molecule as a linear even-numbered chain is taken as an independent variable, given its importance for the crystal structure stability (Thalladi et al., 2000). Our estimation method has then the following form

$$
\begin{aligned}
\Delta H_{\text {fus }}^{\mathrm{est}}\left(T_{\text {fus }}\right)= & a_{1}+a_{2} \tau+a_{3}\left(n_{\mathrm{OH}}+n_{\mathrm{CO}}\right) \\
& +a_{4} n_{\mathrm{COOH}}+a_{5} i_{\text {even }} \\
\Delta S_{\text {fus }}^{\mathrm{est}}\left(T_{\text {fus }}\right)= & a_{1}+a_{2} \tau+a_{3}\left(n_{\mathrm{OH}}+n_{\mathrm{CO}}\right) \\
& +a_{4} n_{\mathrm{COOH}}+a_{5} i_{\mathrm{even}}
\end{aligned}
$$

with $i_{\text {even }}=1$ if the molecule is a linear even-numbered chain and 0 otherwise, and $n_{\mathrm{OH}}, n_{\mathrm{CO}}, n_{\mathrm{COOH}}$ the number of hy- droxy, carbonyl (keto and aldehyde), and acid groups respectively. The fitting equations have the following form:

$$
\begin{aligned}
\Delta S_{\text {fus }, i}^{\exp } & =\frac{\Delta H_{\text {fus }, i}^{\text {est }}}{T_{\text {fus }, i}^{\exp }} \\
\Delta S_{\text {fus }, i}^{\exp } & =\Delta S_{\text {fus }, i}^{\text {est }} \\
0 & =\frac{\Delta H_{\text {fus }, i}^{\text {est }}}{T_{\text {fus }, i}^{\exp }}-\Delta S_{\text {fus }, i}^{\text {est }}
\end{aligned}
$$

where we have used the superscripts "exp" and "est" to clearly distinguish between experimental and estimated values and $i$ refers to data point $i$ (if only $T_{\text {fus, } i}^{\exp }$ is known), or to data couple $i$ (if both $T_{\text {fus }, i}^{\exp }, \Delta H_{\text {fus }, i}^{\exp }$ are known). The last

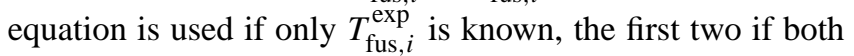
$T_{\text {fus }, i}^{\exp }, \Delta H_{\text {fus }, i}^{\exp }$ are known.

In Table 1 the optimal parameters, obtained by linear regression, are given, as well as the MD, MAD, $R^{2}$, the predicted MD and MAD, and $R_{\mathrm{cv}}^{2}$ (cross-validated $R^{2}$ ), of $\Delta H_{\text {fus }}^{\text {est }}, \Delta S_{\text {fus }}^{\text {est }}$ and $T_{\text {fus }}^{\text {est }}$. Predicted MD, predicted MAD and $R_{\mathrm{cv}}^{2}$ are defined identically as the MD, MAD and $R^{2}$, except that for the estimation of data point (or data couple) $i$, this data point (couple) itself is not used. In this way, the predicted MD, MAD and $R_{\mathrm{cv}}^{2}$ are a measure of the predictive power of the model, while MD, MAD and $R^{2}$ merely show how well the model can fit the observations.

We tried also the following independent variables: cyclicity ( 1 for a cyclic molecule, zero otherwise), and rotational symmetry number (Dannenfelser and Yalkowsky, 1996; Walters and Yalkowsky, 1996) both in its original form and a modified form. In its original form, the rotational symmetry number is the number of indistinguishable positions that can be obtained by rigidly rotating a rigid molecule about its center of mass; for flexible molecules ( $\tau \geq 1)$ it is always unity. In the modified form, the flexibility of the molecule was not considered. None of these independent variables improved the predicted MAD significantly, so they were not retained.

\section{Comparison with estimation methods from the literature}

The methods considered are presented in Table 2. Both the methods of Joback and Reid (1987) (JR) and Marrero and Gani (2001) (MG) are group contribution methods providing both $T_{\text {fus }}(\mathrm{JR}(\mathrm{T}), \mathrm{MG}(\mathrm{T}))$ and $\Delta H_{\text {fus }}(\mathrm{JR}(\mathrm{H}), \mathrm{MG}(\mathrm{H}))$. While the former is relatively simple, the second is a detailed method involving first, second and third order groups. The method of Myrdal and Yalkowsky (1997) (MY) and the more recent variant of Jain et al. (2004a) (JYY) estimate $\Delta S_{\text {fus }}$ from the number of torsional bonds and rotational symmetry of the molecule. The $T_{\text {fus }}$ estimation method of Zhao and Yalkowsky (1999) (ZY) is a composite method: $\Delta S_{\text {fus }}$ 
Table 1. Parameters and statistical diagnostics of the new estimation method.

\begin{tabular}{|c|c|c|c|c|c|c|c|c|c|c|c|}
\hline & $a_{1}$ & $a_{2}$ & $a_{3}$ & $a_{4}$ & $a_{5}$ & MD & MAD & $R^{2}$ & pred. MD & pred. MAD & $R_{\mathrm{cv}}^{2}$ \\
\hline$\frac{\Delta H_{\text {fus }}^{\text {est }}}{\mathrm{kJmol}^{-1}}$ & -12.895 & 1.807 & 11.552 & 15.327 & 12.358 & -0.3 & 5.2 & 0.66 & -0.2 & 6.0 & 0.55 \\
\hline$\frac{\Delta S_{\text {fus }}^{\text {est }}}{\mathrm{JK}^{-1} \mathrm{~mol}^{-1}}$ & -8.508 & 6.520 & 23.890 & 25.379 & 18.153 & -1.0 & 12.7 & 0.62 & -0.7 & 14.7 & 0.51 \\
\hline \multicolumn{6}{|c|}{$T_{\text {fus }} / \mathrm{K}$, from $\Delta H_{\text {fus }}^{\text {est }}, \Delta S_{\text {fus }}^{\text {est }}$} & -4.7 & 24.8 & 0.61 & -4.9 & 26.4 & 0.68 \\
\hline \multicolumn{6}{|c|}{$\omega_{l s}$, from $\Delta H_{\text {fus }}^{\mathrm{est}}, \Delta S_{\text {fus }}^{\text {est }}$} & 0.0 & 0.32 & 0.66 & 0.0 & 0.35 & 0.58 \\
\hline \multicolumn{6}{|c|}{$\omega_{l s}$, from $\Delta H_{\text {fus }}^{\text {est }}, T_{\text {fus }}^{\exp }$} & 0.0 & 0.23 & 0.81 & 0.0 & 0.27 & 0.74 \\
\hline \multicolumn{6}{|c|}{$\omega_{l s}$, from $\Delta S_{\text {fus }}^{\mathrm{est}}, T_{\text {fus }}^{\exp }$} & 0.0 & 0.22 & 0.84 & 0.0 & 0.26 & 0.78 \\
\hline
\end{tabular}

Table 2. Fusion property estimation methods considered in this work.

\begin{tabular}{lll}
\hline Notation & Source & Form \\
\hline $\mathrm{JR}(\mathrm{T})$ & Joback and Reid (1987) & $T_{\text {fus }}^{\mathrm{JR}}=\sum_{i} n_{i} g_{i}$ \\
$\mathrm{JR}(\mathrm{H})$ & Joback and Reid (1987) & $\Delta H_{\text {fus }}^{\mathrm{JR}}=\sum_{i} n_{i} g_{i}$ \\
$\mathrm{MG}(\mathrm{T})$ & Marrero and Gani (2001) & $e^{T_{\text {fus }}^{\mathrm{MG}}=\sum_{i} n_{i} g_{i}}$ \\
$\mathrm{MG}(\mathrm{H})$ & Marrero and Gani (2001) & $\Delta H_{\text {fus }}^{\mathrm{MG}}=\sum_{i} n_{i} g_{i}$ \\
$\mathrm{MY}(\mathrm{S})$ & Myrdal and Yalkowsky (1997) & $\Delta S_{\text {fus }}^{\mathrm{MY}}=g_{1}$ \\
& & $+g_{2} \ln \sigma+g_{3} \tau^{\mathrm{b}}$ \\
$\mathrm{JYY}(\mathrm{S})$ & Jain et al. (2004a) & $\Delta S_{\text {fus }}^{\mathrm{J}}:$ as $\Delta S_{\text {fus }}^{\mathrm{MY}}$ \\
$\mathrm{ZY}(\mathrm{T})$ & Zhao and Yalkowsky (1999) & $T_{\text {fus }}^{\mathrm{ZY}}=\frac{\sum_{i} n_{i} g_{i}}{\Delta S_{\text {fus }}^{\mathrm{MY}}}$ \\
$\mathrm{JYY}(\mathrm{T})$ & Jain et al. (2004b) & $T_{\text {fus }}^{\mathrm{J}}=\frac{\sum_{i} n_{i} g_{i}}{\Delta S_{\text {fus }}^{\mathrm{J}}}$ \\
\hline
\end{tabular}

a $g_{i}$ represent parameter values, $n_{i}$ the frequency of group $i$.

$\mathrm{b}_{\sigma} \sigma$ is the rotational symmetry number (Dannenfelser and Yalkowsky, 1996) and $\tau$ the effective torsional bond number (Myrdal et al., 1996).

is fixed by the MY method $\left(\Delta S_{\text {fus }}^{\mathrm{MY}}\right)$ and $\Delta H_{\text {fus }}$ is written as a sum of group contributions $\sum_{i} n_{i} g_{i}$, with the contributions $g_{i}$ determined by fitting $\left(\sum_{i} n_{i} g_{i}\right) / \Delta S_{\text {fus }}^{\mathrm{MY}}$ to experimental $T_{\text {fus. }}$. The more recent variant of Jain et al. (2004b) (JYY) is derived along the same lines, but with $\Delta S_{\text {fus }}$ fixed by the JYY method.

\subsection{Fusion temperature}

Figure 4 gives estimated vs. experimental $T_{\text {fus }}$ for the methods from the literature and the current method. The JR(T)

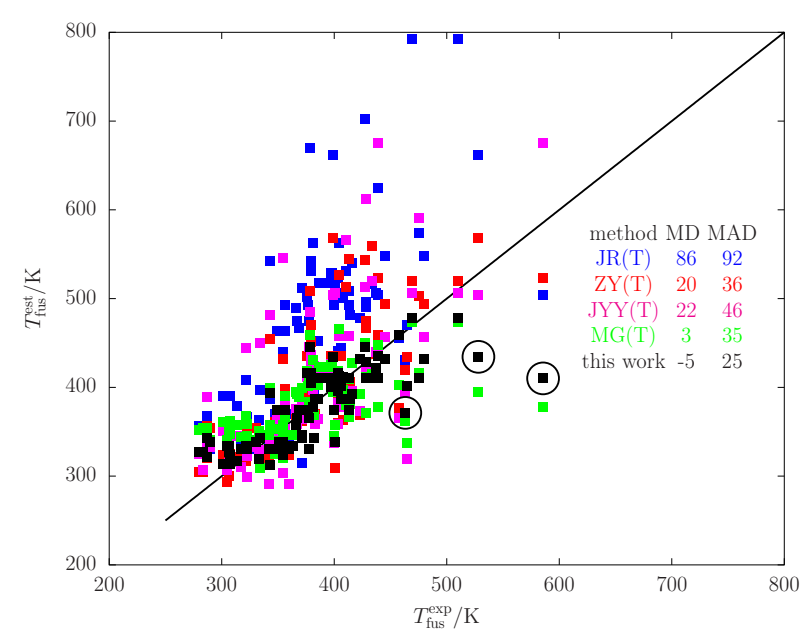

Fig. 4. Estimated vs. experimental $T_{\text {fus }}$ for various literature methods, and the method developed in this work. The MD and MAD of the methods are also given. The 1:1 diagonal is indicated (black line). The three most important outliers of the new method are indicated by a circle (from small to high experimental $T_{\text {fus }}$ : 3-hydroxy2-(hydroxymethyl)-2-methylpropanoic acid, galactaric acid, trans1,4-cyclohexane dicarboxylic acid).

method gives a very large error on the fusion temperature. This can be ascribed to the fact that this method considers $T_{\text {fus }}$ as a sum of group contributions - while it is actually not a group property (Chickos and Nichols, 2001)-, resulting in large overestimations for larger molecules (e.g. $275 \mathrm{~K}$ for citric acid). Clearly, the JR(T) method is not suitable to estimate fusion point of functionalised acids. We note that a similar failure occurs for the estimation of boiling points by the JR method (Stein and Brown, 1994). Of the literature methods, the $\mathrm{ZY}(\mathrm{T})$ and $\mathrm{MG}(\mathrm{T})$ methods perform best for fusion temperature in terms of MAD, but $\mathrm{MG}(\mathrm{T})$ has 

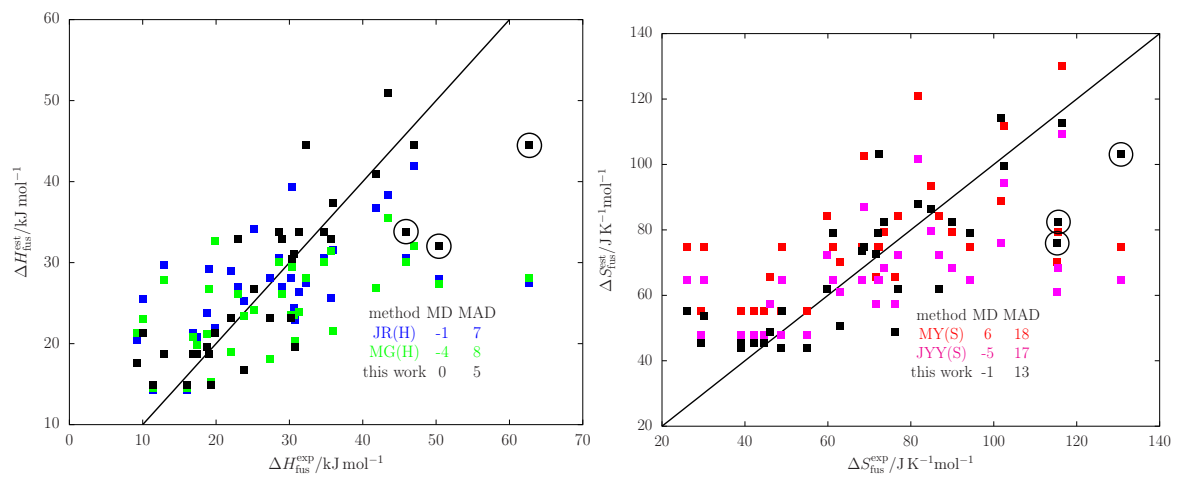

Fig. 5. Estimated vs. experimental $\Delta H_{\text {fus }}$ (left) and $\Delta S_{\text {fus }}$ (right) for various literature methods, and for the method developed in this work. The MD and MAD of the methods are also given. The 1:1 diagonal is indicated (black line). The three most important outliers of the new method are indicated by a circle (from small to high experimental $\Delta H_{\text {fus }}$ : 3-oxoglutaric acid, oxosuccinic acid, tartaric acid).

in addition a low bias. The more recent version of $\mathrm{ZY}(\mathrm{T})$, $\mathrm{JYY}(\mathrm{T})$, actually performs worse. The method developed in this work has a significantly lower error than the literature methods. For all methods, the error increases with $T_{\text {fus. }}$ The most important outliers for the new method as well as for the best performing of the literature methods, MG(T)- are trans-1,4-cyclohexane dicarboxylic diacid, galactaric acid, and 3-hydroxy-2-(hydroxymethyl)-2-methyl propanoic acid. For all three of them the melting point is underestimated. Trans-1,4-cyclohexane dicarboxylic diacid has a much higher melting point than the other cyclic dicarboxylic acids in the dataset. Probably the two acid functionalities at opposite and trans positions in the ring, allow for a crystal structure that is considerably more stable. The melting point of galactaric acid is much higher than that of its stereo-isomer glucaric acid. So also here, a particularly stable crystal structure is the likely explanation of the poorly estimated $T_{\text {fus }}$.

\subsection{Fusion enthalpy and entropy}

Our data set for $\Delta H_{\text {fus }}$ (and hence also for $\Delta S_{\text {fus }}$ ) is considerably smaller than for $T_{\text {fus }}$. For example, the three most important outliers with respect to $T_{\text {fus }}$ (see the Sect. 6.1) are not included.

Notwithstanding its high detail, the $\mathrm{MG}(\mathrm{H})$ method performs worse than the JR(H) method in estimating fusion enthalpy, and has a relatively high bias (Fig. 5). For fusion entropy estimation, the MY(S) and JYY(S) method have a similar precision and bias. The newly developed method shows again the best performance. Most important outliers for the new method, both for $\Delta H_{\text {fus }}$ and $\Delta S_{\text {fus }}$ are oxosuccinic acid, 3-oxoglutaric acid and tartaric acid (the value of Booth et al., 2010), which are in all three cases underestimated. The $\Delta H_{\text {fus }}$ value of tartaric acid of Booth et al. (2010) is almost twice larger than that of d-tartaric acid as present in Acree and Chickos (2010) and NIST. Probably Booth et al. (2010) investigated a different stereo-isomer that forms a more stable crystal structure. 3-oxoglutaric acid has a substantially higher $\Delta H_{\text {fus }}$ value than its isomer 2-oxoglutaric acid, which is again an indication of a particularly stable crystal structure.

\subsection{Estimation of $\omega_{l s}$}

For the estimation of $\omega_{l s}$ (under assumption of $\Delta C_{p, l s}=0$ ), the estimation of two fusion properties is necessary if no experimental fusion data are available. Combinations with $\mathrm{JR}(\mathrm{T})$ give a large positive bias for $\omega_{l s}$ due to the large overestimation of $T_{\text {fus }}$ (Fig. 6). Note that the error increases as $\omega_{l s}$ increases. Oxosuccinic acid and tartaric acid are again outliers. Estimations of $\omega_{l s}$ are much better if the fusion temperature is already known. Of the literature methods, MY(S) and $J Y Y(S)$ in combination with the experimental fusion temperature provide the best results. Table S2 of the Supplement contains the experimental $\omega_{l s}$ and $\omega_{l s}$ estimated using (i) the experimental $T_{\text {fus }}$ and (ii) $\Delta H_{\text {fus }}$ as calculated by the new method.

Again, the current method performs better than the literature methods. This can at least in part be ascribed to the fact that much experimental data used to develop this model is more recent than the methods described in Sect. 6.

\section{Conclusions}

To derive subcooled liquid vapour pressure from solid vapour pressure, knowledge of the fusion properties is necessary. A simple method is developed to estimate the fusion properties of $\mathrm{C}_{2}-\mathrm{C}_{10}$ saturated acids, with one or more other functional groups: hydroxyl, carbonyl, and/or acid. Several fusion property estimation methods from the literature are also tested for these compounds. The newly proposed method has a much narrower scope than the considered literature methods, but has also a significantly smaller error for the target compounds. From the literature methods, best solid-to- 

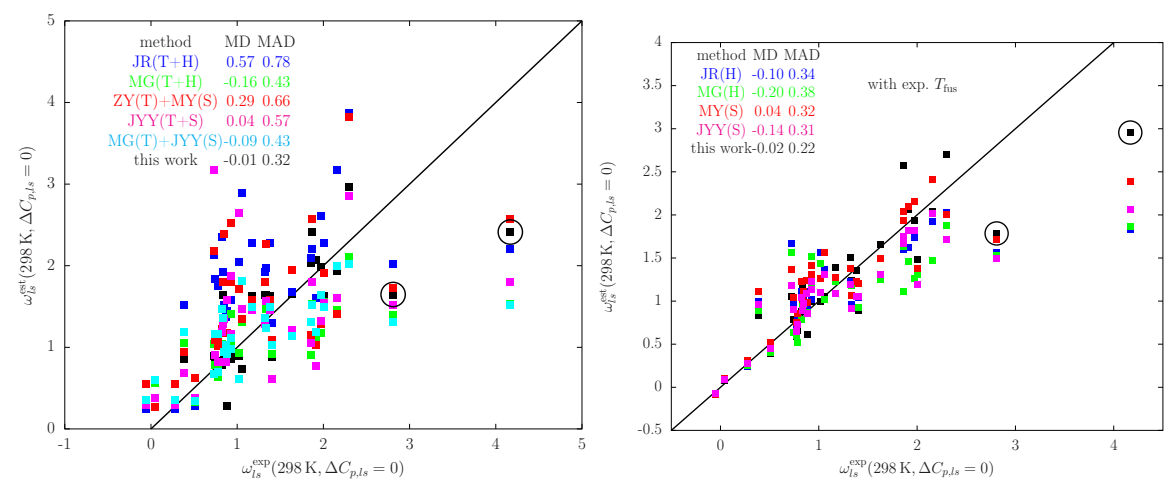

Fig. 6. Estimated vs. experimental $\omega_{l s}$ at $298 \mathrm{~K}$, assuming $\Delta C_{p, l s}=0$. Left: fully estimated. Right: using the experimental $T_{\text {fus }}$, with $\Delta H_{\text {fus }}$ or $\Delta S_{\text {fus }}$ estimated. The MD and MAD of the methods are also given. The 1:1 diagonal is indicated (black line). The two most important outliers of the new method are indicated by a circle (from small to high experimental $\omega_{l s}$ : oxosuccinic acid, tartaric acid).

subcooled liquid conversion factors are obtained by combining the $\Delta S_{\text {fus }}$ estimation method of Myrdal and Yalkowsky (1997) or Jain et al. (2004a) with the experimental fusion temperature or with the estimated fusion temperature using the method of Marrero and Gani (2001) if no experimental fusion temperature is available. The $T_{\text {fus }}$ estimation method of Joback and Reid (1987) should be avoided as it gives gross overestimations for large $T_{\text {fus }}$.

To the best of our knowledge, no data on $\Delta C_{p, l s}$ is available for functionalised acids, although it is potentially important for a correct solid-subcooled liquid conversion factor, especially for compounds with a high $T_{\text {fus }}, \Delta H_{\text {fus }}$. Approximating $\Delta C_{p, l s}$ by $C_{p, l}^{\text {Chickos }}-C_{p, s}^{\text {Chickos }}$ (Chickos, 2003) probably overestimates $\Delta C_{p, l s}$.

The UNIFAC activity coefficient model parameterisations of Peng et al. (2001); Raatikainen and Laaksonen (2005) do not conform with the solubility data of the longer diacids (7-9 carbon atoms) while the original UNIFAC parameterisation, combined with the assumption of $\Delta C_{p, l s}=\Delta S_{\text {fus }}$ performs best for the linear diacids (3-9 carbon atoms). Hence experimental knowledge on $\Delta C_{p, l s}$ can also improve our understanding on non-ideality.

Even with the newly developed method, substantial uncertainty remains. This is probably for a large part due to differences in crystal packings; for several species in our dataset, there are large differences in fusion properties although the molecular connectivities are the same (e.g. glucaric vs. galactaric acid). Our model, like the considered literature methods, does not take stereo-information (e.g. cis or trans isomers) into account. To improve modeling of fusion properties this information should be included. However, this will make the model considerably more complex and moreover, stereo-information is not always available. Another obvious obstacle for the development of better estimation methods is the paucity of experimental fusion data, especially of fusion enthalpies. For example, a fusion enthalpy is available for only five out of 33 compounds in our dataset with one acid functionality. Therefore, more fusion data measurements are certainly desirable.

\section{Supplementary material related to this article is available online at: http://www.atmos-chem-phys.net/11/8385/2011/ acp-11-8385-2011-supplement.pdf.}

Acknowledgements. This work was supported by the projects IBOOT (SD/AT/03B, 2006-2010) and BIOSOA (SD/CS/05A, 2011-2014) funded by the Belgian Science Policy Office.

Edited by: G. McFiggans

\section{References}

Acree, W. J. and Chickos, J. S.: Phase transition enthalpy measurements of organic and organometallic compounds. Sublimation, vaporization and fusion enthalpies from 1880 to 2010, J. Phys. Chem. Ref. Data, 39, 043 101, 1-942, 2010.

Aldrich: Catalog Handbook of Fine Chemicals, Aldrich Chemical Company, Inc., Milwaukee, WI, USA, 1990.

Apelblat, A. and Manzurola, E.: Solubility of oxalic, malonic, succinic, adipic, maleic, malic, citric, and tartaric acids in water from 278.15 to 338.15 K, J. Chem. Thermodyn., 19, 317-320, 1987.

Apelblat, A. and Manzurola, E.: Solubility of suberic, azelaic, levulinic, glycolic, and diglycolic acids in water from $278.25 \mathrm{~K}$ to 361.35 K, J. Chem. Thermodyn., 22, 289-292, 1990.

Arshadi, M. R.: Determination of heats of sublimation of organic compounds by a mass spectrometric-knudsen effusion method, J. Chem. Soc. Faraday T., 1, 1569-1571, 1974.

Baboukas, E. D., Kanakidou, M., and Mihalopoulos, N.: Carboxylic acids in gas and particulate phase above the Atlantic Ocean, J. Geophys. Res., 105, 14459-14472, doi:10.1029/1999JD900977, 2000.

Bilde, M. and Pandis, S. N.: Evaporation rates and vapor pressures of individual aerosol species formed in the atmospheric 
oxidation of $\alpha$ - and $\beta$-pinene, Environ. Sci. Technol., 35, 33443349, 2001.

Bilde, M., Svenningsson, B., Monster, J., and Rosenorn, T.: Evenodd alternation of evaporation rates and vapor pressures of $\mathrm{C}_{3}-$ $\mathrm{C}_{9}$ dicarboxylic acid aerosols, Environ. Sci. Technol., 37, 13711378, 2003.

Booth, A. M., Barley, M. H., Topping, D. O., McFiggans, G., Garforth, A., and Percival, C. J.: Solid state and sub-cooled liquid vapour pressures of substituted dicarboxylic acids using Knudsen Effusion Mass Spectrometry (KEMS) and Differential Scanning Calorimetry, Atmos. Chem. Phys., 10, 4879-4892, doi:10.5194/acp-10-4879-2010, 2010.

Booth, A. M., Montague, W. J., Barley, M. H., Topping, D. O., McFiggans, G., Garforth, A., and Percival, C. J.: Solid state and subcooled liquid vapour pressures of cyclic aliphatic dicarboxylic acids, Atmos. Chem. Phys., 11, 655-665, doi:10.5194/acp-11655-2011, 2011.

Bradley, R. S. and Cotson, S.: The vapour pressure and lattice energy of hydrogen-bonded crystals, Part II, $\alpha$ - and $\beta$-anhydrous oxalic acid and tetragonal pentaerythritol, J. Chem. Soc., 16841688, 1953.

Cappa, C. D., Lovejoy, E. R., and Ravishankara, A. R.: Determination of evaporation rates and vapor pressures of very low volatility compounds: a study of the $\mathrm{C} 4-\mathrm{C} 10$ and $\mathrm{C} 12$ dicarboxylic acids, J. Phys. Chem. A, 111, 3099-3109, 2007.

Cappa, C. D., Lovejoy, E. R., and Ravishankara, A. R.: Evaporation rates and vapor pressures of the even-numbered C8-C18 monocarboxylic acids, J. Phys. Chem. A, 112, 3959-3964, 2008a.

Cappa, C. D., Lovejoy, E. R., and Ravishankara, A. R.: Evidence for liquid-like and nonideal behavior of a mixture of organic aerosol components, P. Natl. Acad. Sci. USA, 105, 18687-18691, 2008b.

Chickos, J. S.: Enthalpies of vaporization of organic and organometallic compounds, 1880-2002, J. Phys. Chem. Ref. Data, 32, 519-878, 2003.

Chickos, J. S. and Nichols, G.: Simple relationships for the estimation of melting temperatures of homologous series, J. Chem. Eng. Data, 46, 562-573, 2001.

Claeys, M., Szmigielski, R., Kourtchev, I., Van der Veken, P., Vermeylen, R., Maenhaut, W., Jaoui, M., Kleindienst, T. E., Lewandowski, M., Offenberg, J. H., and Edney, E. O.: Hydroxydicarboxylic acids: markers for secondary organic aerosol from the photooxidation of alpha-pinene, Environ. Sci. Technol., 41, 1628-1634, 2007.

Dannenfelser, R.-M. and Yalkowsky, S. H.: Estimation of entropy of melting from molecular structure: a non-group contribution method, Ind. Eng. Chem. Res., 35, 1483-1486, 1996.

Fredenslund, A., Jones, R. L., and Prausnitz, J. M.: Groupcontribution estimation of activity-coefficients in nonideal liquid-mixtures, AIChE J., 21, 1086-1099, 1975.

Frosch, M., Zardini, A. A., Platt, S. M., Müller, L., Reinnig, M.C., Hoffmann, T., and Bilde, M.: Thermodynamic properties and cloud droplet activation of a series of oxo-acids, Atmos. Chem. Phys., 10, 5873-5890, doi:10.5194/acp-10-5873-2010, 2010.

Grant, D., Mehdizadeh, M., Chow, A.-L., and Fairbrother, J.: Nonlinear van't Hoff solubility-temperature plots and their pharmaceutical interpretation, Int. J. Pharm., 18, 25-38, 1984.

Hansen, H. K., Rasmussen, P., Fredenslund, A., Schiller, M., and Gmehling, J.: Vapor-liquid-equilibria by unifac group contribution, 5, Revision and extension, Ind. Eng. Chem. Res., 30, 2352-
2355, 1991.

Jain, A., Yang, G., and Yalkowsky, S. H.: Estimation of total entropy of melting of organic compounds, Ind. Eng. Chem. Res., 43, 4376-4379, 2004a.

Jain, A., Yang, G., and Yalkowsky, S. H.: Estimation of melting points of organic compounds, Ind. Eng. Chem. Res., 43, 76187621, 2004b.

Joback, K. and Reid, R.: Estimation of pure-component properties from group-contributions, Chem. Eng. Commun., 57, 233-243, 1987.

Koponen, I. K., Riipinen, I., Hienola, A., Kulmala, M., and Bilde, M.: Thermodynamic properties of malonic, succinic, and glutaric acids: evaporation rates and saturation vapor pressures, Environ. Sci. Technol., 41, 3926-3933, 2007.

Lide, D. R. (Ed.): CRC Handbook of Chemistry and Physics, CRC Press, 80 edn., 2000.

Limbeck, A., Puxbaum, H., Otter, L., and Scholes, M. C.: Semivolatile behavior of dicarboxylic acids and other polar organic species at a rural background site (Nylsvley, RSA), Atmos. Environ., 35, 1853-1862, 2001.

Linstrom, P. and Mallard, W. (Eds.): NIST Chemistry WebBook, NIST Standard Reference Database Number 69, National Institute of Standards and Technology, Gaithersburg MD, 20899, http://webbook.nist.gov/chemistry/, last access: 2010.

Marrero, J. and Gani, R.: Group-contribution based estimation of pure component properties, Fluid Phase Equilibr., 183/184, 183208, 2001.

Monster, J., Rosenorn, T., Svenningsson, B., and Bilde, M.: Evaporation of methyl- and dimethyl-substituted malonic, succinic, glutaric and adipic acid particles at ambient temperatures, J. Aerosol Sci., 35, 1453-1465, 2004.

Myrdal, P. B. and Yalkowsky, S. H.: Estimating pure component vapor pressures of complex organic molecules, Ind. Eng. Chem. Res., 36, 2494-2499, 1997.

Myrdal, P. B., Krzyzaniak, J. F., and Yalkowsky, S. H.: Modified Trouton's rule for predicting the entropy of boiling, Ind. Eng. Chem. Res., 35, 1788-1792, 1996.

Neau, S. H., Bhandarkar, S. V., and Hellmuth, E. W.: Differential molar heat capacities to test ideal solubility estimations, Pharm. Res., 14, 601-605, 1997.

Peng, C., Chan, M. N., and Chan, C. K.: The hygroscopic properties of dicarboxylic and multifunctional acids: measurements and UNIFAC predictions, Environ. Sci. Technol., 35, 4495-4501, 2001.

Pope, F. D., Tong, H.-J., Dennis-Smither, B. J., Griffiths, P. T., Clegg, S. L., Reid, J. P., and Cox, R. A.: Studies of single aerosol particles containing malonic acid, glutaric acid, and their mixtures with sodium chloride, II, Liquid-state vapor pressures of the acids, J. Phys. Chem. A, 114, 10156-10165, 2010.

Prausnitz, J. M., Lichtenthaler, R. N., and de Azevedo, E. G.: Molecular Thermodynamics of Fluid-Phase Equilibria, 3rd edn., Prentice-Hall PTR, Upper Saddle River, New Jersey, 07458, 1999.

Raatikainen, T. and Laaksonen, A.: Application of several activity coefficient models to water-organic-electrolyte aerosols of atmospheric interest, Atmos. Chem. Phys., 5, 2475-2495, 2005, http://www.atmos-chem-phys.net/5/2475/2005/.

Ribeiro da Silva, M. A. V., Monte, M. J. S., and Ribeiro, J. R.: Standard enthalpies, entropies, and Gibbs functions of sublima- 
tion of four alkyl-substituted malonic acids, J. Chem. Eng. Data, 45, 756-759, 2000.

Ribeiro da Silva, M. A. V., Monte, M. J. S., and Ribeiro, J. R.: Thermodynamic study on the sublimation of succinic acid and of methyl- and dimethyl-substituted succinic and glutaric acids, J. Chem. Thermodyn., 33, 23-31, 2001.

Riipinen, I., Svenningsson, B., Bilde, M., Gaman, A., Lehtinen, K., and Kulmala, M.: A method for determining thermophysical properties of organic material in aqueous solutions: Succinic acid, Atmos. Res., 82, 579-590, 2006.

Riipinen, I., Koponen, I., Frank, G., Hyvarinen, A.-P., Vanhanen, J., Lihavainen, H., Lehtinen, K., Bilde, M., and Kulmala, M.: Adipic and malonic acid aqueous solutions: surface tensions and saturation vapor pressures, J. Phys. Chem. A, 111, 12995-13002, 2007.

Roux, M. V., Temprado, M., and Chickos, J. S.: Vaporization, fusion and sublimation enthalpies of the dicarboxylic acids from $\mathrm{C}_{4}$ to $\mathrm{C}_{14}$ and $\mathrm{C}_{16}$, J. Chem. Thermodyn., 37, 941-953, 2005.

Salo, K., Jonsson, Å. M., Andersson, P. U., and Hallquist, M.: Aerosol volatility and enthalpy of sublimation of carboxylic acids, J. Phys. Chem. A, 114, 4586-4594, 2010.

Soonsin, V., Zardini, A. A., Marcolli, C., Zuend, A., and Krieger, U. K.: The vapor pressures and activities of dicarboxylic acids reconsidered: the impact of the physical state of the aerosol, Atmos. Chem. Phys., 10, 11753-11767, doi:10.5194/acp-1011753-2010, 2010.
Stein, S. E. and Brown, R. L.: Estimation of normal boiling points from group contributions, J. Chem. Inf. Comp. Sci., 34, 581-587, 1994.

Thalladi, V. R., Nüsse, M., and Boese, R.: The melting point alternation in $\alpha, \omega$-alkanedicarboxylic acids, J. Am. Chem. Soc., 122, 9227-9236, 2000.

Tsonopoulos, C.: Properties of Dilute Aqueous Solutions of Organic Solutes, Ph.D. thesis, University of California, CA, USA, 1970.

Walters, W. P. and Yalkowsky, S. H.: ESCHERA Computer Program for the Determination of External Rotational Symmetry Numbers from Molecular Topology, J. Chem. Inf. Comput. Sci., 36, 1015-1017, 1996.

Yu, J., Cocker, D. R., Griffin, R. J., Flagan, R. C., and Seinfeld, J. H.: Gas-phase ozone oxidation of monoterpenes: gaseous and particulate products, J. Atmos. Chem., 34, 207-258, 1999.

Zardini, A. A., Krieger, U. K., and Marcolli, C.: White light Mie resonance spectroscopy used to measure very low vapor pressures of substances in aqueous solution aerosol particles, Opt. Express, 14, 6951-6962, 2006.

Zhao, L. and Yalkowsky, S. H.: A combined group contribution and molecular geometry approach for predicting melting points of aliphatic compounds, Ind. Eng. Chem. Res., 38, 3581-3584, 1999. 